Jennifer Alexandra Mendez Rangel

\title{
Sobre a Interferência Produzida por Estações \\ Terrenas a Bordo de Embarcações em Receptores do Serviço Fixo Terrestre
}

Dissertação apresentada como requisito parcial para obtenção do grau de Mestre pelo Programa de Pós-graduação em Engenharia Elétrica do Departamento de Engenharia Elétrica da PUC-Rio.

Orientador: Prof. José Mauro Pedro Fortes 


\title{
Jennifer Alexandra Mendez Rangel
}

\section{Sobre a Interferência Produzida por Estações Terrenas a Bordo de Embarcações em Receptores do Serviço Fixo Terrestre}

\begin{abstract}
Dissertação de Mestrado apresentada como requisito parcial para obtenção do grau de Mestre pelo Programa de Pósgraduação em Engenharia Elétrica do Departamento de Engenharia Elétrica do Centro Técnico Científico da PUC-Rio. Aprovada pela Comissão Examinadora abaixo assinada.
\end{abstract}

Prof. José Mauro Pedro Fortes

Orientador

Centro de Estudos em Telecomunicações - PUC-Rio

Prof. Raimundo Sampaio Neto

Centro de Estudos em Telecomunicações - PUC-Rio

Prof. Paulo Roberto Rosa Lopes Nunes

IME

Prof. Rodrigo Caiado de Lamare

Centro de Estudos em Telecomunicações - PUC-Rio

Prof. José Eugênio Leal Coordenador Setorial do Centro Técnico Cientifico - PUC-Rio 
Todos os direitos reservados. É proibida a reprodução total ou parcial do trabalho sem autorização da universidade, do autor e do orientador.

\section{Jennifer Alexandra Mendez Rangel}

Graduou-se em Engenharia de Telecomunicações na Universidad de San Buenaventura (Bogotá, Colômbia).

Ficha Catalográfica

Méndez Rangel, Jennifer Alexandra

Sobre a Interferência Produzida por Estações Terrenas a Bordo de Embarcações em Receptores do Serviço Fixo Terrestre/ Jennifer Alexandra Mendez Rangel; orientador: José Mauro Pedro Fortes. - Rio de Janeiro : PUC-Rio, Departamento de Engenharia Elétrica, 2014.

84 f: il.(color.) ; $30 \mathrm{~cm}$

1. Dissertação (mestrado) - Pontifícia Universidade Católica do Rio de Janeiro, Departamento de Engenharia Elétrica.

Inclui referências bibliográficas.

1. Engenharia Elétrica - Tese. 2. Sistemas Via Satélite. 3. Estações terrenas a bordo de embarcações I. Fortes, José Mauro. II. Pontifícia Universidade Católica do Rio de Janeiro. Departamento de Engenharia Elétrica. III. Título. 
Siempre he pensado que las dedicatorias son injustas... por más de que se quiera plasmar en un puñado de letras el amor hacia las personas por las que uno hace las cosas es casi imposible. Todos los que hacen parte de mi motivación lo saben y saben que los amo. Esta vez quiero dedicarle este logro a mi fan número uno, a ese que tristemente, no está más para leer estas letras, pero que sabe que vive en mi corazón. 


\section{Agradecimentos}

A Deus e sua sábia vontade, por tudo o que eu sou, por meu passado, meu presente e meu futuro.

A minha família, sem dúvida, que tem me oferecido sempre, e me oferecerão por toda a vida, seu infinito amor. Obrigada especial a Lucas, Alicia, Catherine e Paula por serem a minha grande motivação, porque tudo foi obtido como uma vitória conjunta, resultado de todos os ensinamentos e do apoio que vocês têm me irradiado ao longo dos anos.

A PUC-Rio, ao seu corpo docente, a direção e a administração da secretaria, por abrir-me as suas portas e deixar-me crescer academicamente, concedendo-me uma excelente formação da mais alta qualidade. Da mesma forma agradeço o apoio financeiro provido pela CAPES, oferecendo grandes oportunidades aos profissionais que desejam especializar-se e qualificar-se na formação de nível superior.

A meu orientador e excelente professor Jose Mauro P. Fortes, por acreditar no meu trabalho e nas minhas habilidades, orientando-me de forma brilhante, por que cada minuto que trabalhei com ele, aprendi muito e espero continuar fazendo-o.

Ao Professor Emanoel Paiva de Oliveira Costa que desinteressadamente nos ajudou e guiou com seu amplo conhecimento e com sua imensa experiência nos tópicos de pesquisa envolvidos.

Ao Professor Carlos Lozano Garzón, outro excelente orientador que a vida me outorgou, quem me guiou durante minha formação. Todos os sonhos têm um começo e neste começo ele esteve presente, me encorajando e alimentando diariamente o meu desejo de sobressair.

A Mauricio Nava, graças a um cruzamento de rotas no momento perfeito, tem sido meu suporte na cidade maravilhosa e na minha vida, meus sinceros agradecimentos por acreditar cegamente em mim.

Alberth Tamo e Vanessa Gonzáles que, nestes dois anos, deram-me ajuda e carinho incondicionais.

A todas as pessoas que encontrei no caminho, entre eles compatriotas, colegas e companheiros do CETUC, pela ajuda, pelos conselhos e pela amizade que me fizeram alcançar este objetivo de vida. 


\section{Resumo}

Méndez Rangel, Jennifer Alexandra; Fortes, José Mauro. Sobre a Interferência Produzida por Estações Terrenas a Bordo de Embarcações em Receptores do Serviço Fixo Terrestre. Rio de Janeiro, 2014. 84p. Dissertação de Mestrado - Departamento de Engenharia Elétrica, Pontifícia Universidade Católica do Rio de Janeiro.

A partir do final dos anos 90, a utilização de estações terrenas a bordo de embarcações (ESVs) tornou-se usual. Estas estações encontram-se instaladas numa variedade de navios e em plataformas móveis que operam em todas as regiões do globo. Em geral, elas operam utilizando o segmento espacial do serviço fixo por satélite (FSS) em partes das bandas $\mathrm{C}$ e $\mathrm{Ku}$ e, muitas vezes, compartilham faixas de frequência com o Serviço Fixo Terrestre (FS). Com base em resultados dos estudos realizados no período 2000-2003, a Conferência Mundial de Radiocomunicações de 2003 (WRC'03) avaliou as condições sob as quais ESVs poderiam operar nas redes FSS sem produzir interferências inaceitáveis aos serviços de radiocomunicações operando de acordo com o Regulamento como, por exemplo, o FS. Esta avaliação resultou numa resolução que estabelece provisões técnicas e regulamentares para a operação de estações terrenas a bordo de embarcações operando em faixas das bandas C e Ku. Esta resolução impõe duas limitações à operação das ESVs: (i) sua distância à costa marítima e (ii) a densidade de e.i.r.p. transmitida na direção do horizonte. Estudos posteriores indicaram ser mais adequada a imposição de restrições que tivessem como base a densidade de fluxo de potência produzida pela ESV em receptores do FS localizados na costa, o que permitiria a operação das ESVs mesmo com distâncias à costa inferiores à distância mínima estabelecida pela resolução da WRC'03. Este fato motivou a proposição de metodologias para determinar novas distâncias mínimas à costa que permitissem a operação das ESVs sem prejuízo dos enlaces terrestres. No presente trabalho, um procedimento baseado numa destas metodologias é utilizado para determinar as distâncias minimas de operação em relação à costa marítima brasileira. Este procedimento permite determinar as distâncias à costa brasileira em função da posição orbital do satélite utilizado e da densidade de e.i.r.p. transmitida pela ESV em direção ao satélite.

\section{Palavras-chave}

Interferência; Estações terrenas a bordo de embarcações; Receptores do serviço fixo terrestre; Critério de densidade de fluxo de potência. 


\section{Abstract}

Méndez Rangel, Jennifer Alexandra; Fortes, José Mauro(Advisor). On the interference produced by Earth Stations on-board Vessels (ESVs) on terrestrial fixed service receivers . Rio de Janeiro, 2014. 84p. MSc. Dissertation - Departamento de Engenharia Elétrica, Pontifícia Universidade Católica do Rio de Janeiro.

Since the end of the '90s the use of Earth Stations on board Vessels (ESVs) became usual. These stations are installed in variety of ships and mobile platforms operating in all regions of the world. Generally they operate using the space segment of Fixed Satellite Service (FSS) in either the C or the $\mathrm{Ku}$ bands, and usually share frequencies with terrestrial Fixed Service systems (FS). Based on results of studies that were conducted in the period 2000-2003 the 2003 World Radiocommunication Conference (WRC'03) evaluated the conditions under which ESVs could operate in FSS networks without producing unacceptable interference to radiocommunications services operating under the Radio Regulations as, for example, FS systems. Based on this assessment, the WRC03 produced a resolution containing technical and operational provisions for ESVs transmitting in the $\mathrm{C}$ and $\mathrm{Ku}$ bands. This resolution imposes two constraints on the operation of the ESVs: a minimum operating distances from the low-water mark and, a maximum value for the e.i.r.p spectral density toward the horizon. Later studies have indicated that constraints based on the power flux density produced by ESV at the FS receiver located at the coastline could be more appropriate and flexible. This approach would allow for the operation of ESVs at closer distances than those established by the WRC'03 resolution. This fact has motivated studies that proposed methodologies do determine new minimum ESV distances to the coast. In the present work, a procedure based on one of these methodologies is used to determine minimal ESV operating distances to the Brazilian coast as a function of orbital position of the satellite being used and of the e.i.r.p. density transmitted by the ESV towards the satellite.

\section{Keywords}

Interference; Earth Stations on-board Vessels (ESVs); terrestrial fixed service receivers; power flux density criterion. 


\section{Sumário}

1 Introdução 13

2 Descrição do Problema e Modelagem Matemática $\quad 15$

2.1 Descrição do Problema 15

2.2 Cálculo do valor pfd no receptor de FS 16

2.3 Metodologia para a determinação da distância mínima à costa 20

3 Resultados Numéricos $\quad 27$

3.1 Distâncias mínimas para ESVs operando na Banda C (5925-6425 MHz) 29

3.2 Distâncias mínimas para ESVs operando na Banda Ku $(14-14,5$ GHz) 51

4 Conclusões $\quad 69$

A Procedimento para determinar o valor $L_{p}$ da perda de propagação que não é excedido durante $p \%$ do tempo 


\section{Lista de figuras}

2.1 Interferência de ESV em um receptor do Serviço Fixo Terrestre. $\quad 17$

2.2 Posicionamento inicial da ESV e do receptor FS 20

2.3 Variação da posição da ESV entorno do ponto C 22

2.4 Variação da posição da ESV entorno do ponto C' 23

2.5 Múltiplos receptores FS 24

2.6 Distância mínima baseada em pfd 24

2.7 Distâncias mínimas baseadas em pfd para diferentes valores de densidade de e.i.r.p 25

2.8 Ilustração da equivalência entre as condições em (2-20) e (2-22). 26

3.1 Limite geográfico baseado em pfd correspondente a perda de propagação de curto prazo. Posição orbital: $103,35^{\circ} \mathrm{W}$, Ponto B: $\left(34,827^{\circ} \mathrm{W} ; 7,986^{\circ} \mathrm{S}\right)$, Ponto C: $\left(32,108^{\circ} \mathrm{W} ; 8,118^{\circ} \mathrm{S}\right)$.

3.2 Limites geográficos baseados em pfd correspondentes a perdas de propagação de curto prazo. Posição orbital: $103,35^{\circ} \mathrm{W}$, Ponto B: $\left(34,827^{\circ} \mathrm{W} ; 7,986^{\circ} \mathrm{S}\right)$, Ponto C: $\left(32,108^{\circ} \mathrm{W} ; 8,118^{\circ} \mathrm{S}\right)$.

3.3 Limites geográficos baseados em pfd correspondentes a perdas de propagação de curto prazo. Posição orbital: $90^{\circ} \mathrm{W}$, Ponto B: $\left(34,827^{\circ} \mathrm{W} ; 7,986^{\circ} \mathrm{S}\right)$, Ponto $\mathrm{C}:\left(32,113^{\circ} \mathrm{W} ; 8,235^{\circ} \mathrm{S}\right)$.

3.4 Limites geográficos baseados em pfd correspondentes a perdas de propagação de curto prazo. Posição orbital: $73^{\circ} \mathrm{W}$, Ponto B: $\left(34,827^{\circ} \mathrm{W} ; 7,986^{\circ} \mathrm{S}\right)$, Ponto C: $\left(32,147^{\circ} \mathrm{W} ; 8,445^{\circ} \mathrm{S}\right)$.

3.5 Limites geográficos baseados em pfd correspondentes a perdas de propagação de curto prazo. Posição orbital: $50^{\circ} \mathrm{W}$, Ponto B: (52, $\left.997^{\circ} \mathrm{W} ; 33,455^{\circ} \mathrm{S}\right)$, Ponto C: $\left(53,317^{\circ} \mathrm{W} ; 36,138^{\circ} \mathrm{S}\right)$.

3.6 Limites geográficos baseados em pfd correspondentes a perdas de propagação de curto prazo. Densidade de e.i.r.p $=53 \mathrm{~dB}[\mathrm{~W} / \mathrm{MHz}] 37$

3.7 Limites geográficos baseados em pfd correspondentes a perdas de propagação de curto prazo. Densidade de e.i.r.p $=45 \mathrm{~dB}[\mathrm{~W} / \mathrm{MHz}] 38$

3.8 Limites geográficos baseados em pfd correspondentes a perdas de propagação de curto prazo. Densidade de e.i.r.p $=35 \mathrm{~dB}[\mathrm{~W} / \mathrm{MHz}] \quad 39$

3.9 Limites geográficos baseados em pfd correspondentes a perdas de propagação de longo prazo. Posição orbital: $103,35^{\circ} \mathrm{W}$, Ponto B: $\left(34,827^{\circ} \mathrm{W} ; 7,986^{\circ} \mathrm{S}\right)$, Ponto C: $\left(32,108^{\circ} \mathrm{W} ; 8,118^{\circ} \mathrm{S}\right)$.

3.10 Limites geográficos baseados em pfd correspondentes a perdas de propagação de longo prazo. Posição orbital: $90^{\circ} \mathrm{W}$, Ponto B: $\left(34,827^{\circ} \mathrm{W} ; 7,986^{\circ} \mathrm{S}\right)$, Ponto C: $\left(32,116^{\circ} \mathrm{W} ; 8,235^{\circ} \mathrm{S}\right)$.

3.11 Limites geográficos baseados em pfd correspondentes a perdas de propagação de longo prazo. Posição orbital: $73^{\circ} \mathrm{W}$, Ponto B: $\left(34,827^{\circ} \mathrm{W} ; 7,986^{\circ} \mathrm{S}\right)$, Ponto C: $\left(32,143^{\circ} \mathrm{W} ; 8,445^{\circ} \mathrm{S}\right)$.

3.12 Limites geográficos baseados em pfd correspondentes a perdas de propagação de longo prazo. Posição orbital: $50^{\circ} \mathrm{W}$, Ponto B: (52, $\left.997^{\circ} \mathrm{W} ; 33,455^{\circ} \mathrm{S}\right)$, Ponto C: $\left(53,317^{\circ} \mathrm{W} ; 36,138^{\circ} \mathrm{S}\right)$.

3.13 Limites geográficos baseados em pfd correspondentes a perdas de propagação de longo prazo. Densidade de e.i.r.p $=53 \mathrm{~dB}[\mathrm{~W} / \mathrm{MHz}] \quad 46$ 
3.14 Limites geográficos baseados em pfd correspondentes a perdas de propagação de longo prazo. Densidade de e.i.r.p $=45 \mathrm{~dB}[\mathrm{~W} / \mathrm{MHz}] \quad 47$

3.15 Limites geográficos baseados em pfd correspondentes a perdas de propagação de longo prazo. Densidade de e.i.r.p $=35 \mathrm{~dB}[\mathrm{~W} / \mathrm{MHz}] \quad 48$

3.16 Limites geográficos baseados em pfd correspondentes a perdas de propagação de longo e curto prazo. Densidade de e.i.r.p $=53 \mathrm{~dB}[\mathrm{~W} / \mathrm{MHz}]$ e posição orbital de $103,35^{\circ} \mathrm{W}$.

3.17 Ilustração da condição (2-22) para dois valores de $\mathrm{p}$ (curto prazo $p / 100=0.000779$ e longo prazo $p / 100=0.02)$ correspondentes ao caso de ESVs operando na Banda C.

3.18 Limite geográfico baseado em pfd correspondente a perda de propagação de curto prazo. Posição orbital: $104,95^{\circ} \mathrm{W}$, Ponto B: $\left(34,827^{\circ} \mathrm{W} ; 7,986^{\circ} \mathrm{S}\right)$, Ponto $\mathrm{C}:\left(33,694^{\circ} \mathrm{W} ; 8,043^{\circ} \mathrm{S}\right)$.

3.19 Limite geográfico baseado em pfd correspondente a perda de propagação de curto prazo. Posição orbital: $90^{\circ} \mathrm{W}$, Ponto B: $\left(34,827^{\circ} \mathrm{W} ; 7,986^{\circ} \mathrm{S}\right)$, Ponto $\mathrm{C}:\left(33,697^{\circ} \mathrm{W} ; 8,092^{\circ} \mathrm{S}\right)$.

3.20 Limite geográfico baseado em pfd correspondente a perda de propagação de curto prazo. Posição orbital: $73^{\circ} \mathrm{W}$, Ponto B: $\left(34,827^{\circ} \mathrm{W} ; 7,986^{\circ} \mathrm{S}\right)$, Ponto $\mathrm{C}:\left(33,709^{\circ} \mathrm{W} ; 8,179^{\circ} \mathrm{S}\right)$.

3.21 Limite geográfico baseado em pfd correspondente a perda de propagação de curto prazo. Posição orbital: $50^{\circ} \mathrm{W}$, Ponto B: (52, $\left.997^{\circ} \mathrm{W} ; 33,455^{\circ} \mathrm{S}\right)$, Ponto C: $\left(53,128^{\circ} \mathrm{W} ; 34,574^{\circ} \mathrm{S}\right)$.

3.22 Limites geográficos baseados em pfd correspondentes a perdas de propagação de curto prazo. Densidade de e.i.r.p $=47,7 \mathrm{~dB}[\mathrm{~W} / \mathrm{MHz}] 57$

3.23 Limites geográficos baseados em pfd correspondentes a perdas de propagação de curto prazo. Densidade de e.i.r.p $=45 \mathrm{~dB}[\mathrm{~W} / \mathrm{MHz}] 58$

3.24 Limites geográficos baseados em pfd correspondentes a perdas de propagação de curto prazo. Densidade de e.i.r.p $=35 \mathrm{~dB}[\mathrm{~W} / \mathrm{MHz}] \quad 59$

3.25 Limite geográfico baseado em pfd correspondente a perda de propagação de longo prazo. Posição orbital: $104,95^{\circ} \mathrm{W}$, Ponto B: (34, $\left.827^{\circ} \mathrm{W} ; 7,986^{\circ} \mathrm{S}\right)$, Ponto C: $\left(33,694^{\circ} \mathrm{W} ; 8,043^{\circ} \mathrm{S}\right)$.

3.26 Limite geográfico baseado em pfd correspondente a perda de propagação de longo prazo. Posição orbital: $90^{\circ} \mathrm{W}$, Ponto B: $\left(34,827^{\circ} \mathrm{W} ; 7,986^{\circ} \mathrm{S}\right)$, Ponto C: $\left(33,697^{\circ} \mathrm{W} ; 8,092^{\circ} \mathrm{S}\right)$.

3.27 Limite geográfico baseado em pfd correspondente a perda de propagação de longo prazo. Posição orbital: $73^{\circ} \mathrm{W}$, Ponto B: $\left(34,827^{\circ} \mathrm{W} ; 7,986^{\circ} \mathrm{S}\right)$, Ponto $\mathrm{C}:\left(33,709^{\circ} \mathrm{W} ; 8,179^{\circ} \mathrm{S}\right)$.

3.28 Limite geográfico baseado em pfd correspondente a perda de propagação de longo prazo. Posição orbital: $50^{\circ} \mathrm{W}$, Ponto B: $\left(52,997^{\circ} \mathrm{W} ; 33,455^{\circ} \mathrm{S}\right)$, Ponto C: $\left(53,127^{\circ} \mathrm{W} ;-34,574^{\circ} \mathrm{S}\right)$.

3.29 Limites geográficos baseados em pfd correspondentes a perdas de propagação de longo prazo. Densidade de e.i.r.p $=47,7 \mathrm{~dB}[\mathrm{~W} / \mathrm{MHz}] 65$

3.30 Limites geográficos baseados em pfd correspondentes a perdas de propagação de longo prazo. Densidade de e.i.r.p $=40 \mathrm{~dB}[\mathrm{~W} / \mathrm{MHz}] \quad 66$

3.31 Limites geográficos baseados em pfd correspondentes a perdas de propagação de longo prazo. Densidade de e.i.r.p $=35 \mathrm{~dB}[\mathrm{~W} / \mathrm{MHz}] 67$

A.1 Metodologias oferecidas na Recomendação ITU-R P.452 75

A.2 Etapas de cálculo da metodologia 1 da Recomendação ITU-R P. 45276 
A.3 Valor médio anual de $\Delta N[10] \quad 78$

A.4 Refratividade da superfície ao nível do mar $N_{0}[10] \quad 78$

A.5 Exemplo de um perfil de percurso transhorizonte $\quad 80$

A.6 Bloco do modelo de propagação correspondente a Difração $\quad 83$

A.7 Sub-bloco 1 correspondete ao bloco de Difração 83

A.8 Sub-bloco 2 correspondete ao bloco de Difração 84 


\section{Lista de tabelas}

2.1 Limitações técnicas para ESVs transmitindo nas faixas de 5925$6425 \mathrm{MHz}$ e $14-14.5 \mathrm{GHz}$

3.1 Parâmetros utilizados no cálculo da perda de propagação - Banda C 29

3.2 Parâmetros utilizados no cálculo da perda de propagação para Banda Ku 